Bull. Soc. math. France

130 (1), 2002, p. $71-85$

\title{
CÔNE NORMAL ET RÉGULARITÉS DE KUO-VERDIER
}

\author{
par Patrice OrRo \& David Trotman
}

\begin{abstract}
RÉSumÉ. - Nous introduisons de nouvelles régularités de Kuo-Verdier $\left(r^{e}\right)$ et montrons que pour une stratification $C^{2}\left(a+r^{e}\right)$-régulière, en particulier $(w)$-régulière, la fibre du cône normal le long d'une strate $Y$ est égale au cône tangent à la fibre d'une rétraction sur $Y$. Ceci généralise le résultat analogue pour les stratifications sous-analytiques (b)-régulières démontré par J.-P. Henry et M. Merle [9], et aussi le résultat analogue pour les stratifications différentiables $(w+\delta)$-régulières démontré par nous-même [17]. Nous démontrons aussi l'ouverture de la projection du cône normal - appelée pseudo-platitude normale.

Abstract (Normal cone and Kuo-Verdier regularities). - We introduce new KuoVerdier regularities $\left(r^{e}\right)$ and prove that for an $\left(a+r^{e}\right)$-regular (in particular for a $(w)$-regular) $C^{2}$ stratification, the fibre of the normal cone along a stratum $Y$ is equal to the tangent cone of the fibre of a retraction onto $Y$. This generalises the analogous result for (b)-regular subanalytic stratifications proved by J.-P. Henry and M. Merle [9], and also the analogous result for $(w+\delta)$-regular differentiable stratifications proved by the authors [17]. We further prove that the projection of the normal cone is open one says then that the stratification is normally pseudo-flat.
\end{abstract}

Texte reçu le 24 octobre 2000, révisé le 19 février 2001, accepté le 11 avril 2001 Patrice Orro, Laboratoire de Mathématiques (UMR 5127), Université de Savoie, Campus scientifique, 73376 Le Bourget-du-Lac Cedex (France) • E-mail : orro@univsavoie.fr

David Trotman, Laboratoire d'Analyse, Topologie et Probabilités (UMR 6632), Université de Provence, Centre de Mathématiques et Informatique, 13453 Marseille (France)

E-mail : trotman@gyptis.univ-mrs.fr

Classification mathématique par sujets (2000). - 58A35, 32S15.

Mots clefs. - Régularité de Kuo-Verdier, stratification, cône normal, pseudo-platitude normale.

Ce travail a été effectué avec l'aide du CNRS, du JSPS (Japanese Society for the Promotion of Science), et de l'Institut Isaac Newton de l'Université de Cambridge. 


\section{Introduction}

Dans la suite, $k$ est un entier supérieur ou égal à 2 . Soit $\mathcal{Z}$ un fermé stratifié de $\mathbb{R}^{n}$, ayant pour strates des sous-variétés différentiables de classe $C^{k}$. Pour chaque strate $Y$ de $\mathcal{Z}$ on notera $C_{Y} \mathcal{Z}$ le cône normal de $\mathcal{Z}$ le long de $Y$, c'està-dire la restriction au-dessus de $Y$ de l'adhérence de l'ensemble

$$
\{(x, \mu(x \pi(x))): x \in \mathcal{Z}-Y\} \subset \mathbb{R}^{n} \times S^{n-1},
$$

où $\pi$ est la projection canonique locale sur $Y$, et $\mu(x)$ le vecteur unitaire $x /\|x\|$. En fait $C_{Y} \mathcal{Z}$ est la réunion des cônes normaux $C_{Y} X_{i}$, où les $X_{i}$ sont les strates de $\mathcal{Z}$ adhérentes à $Y$.

L'objet principal de cette note est de préciser sous quelles hypothèses sur la stratification $\mathcal{Z}$ les conditions suivantes sont vérifiées :

Condition $(n)$. - La fibre $\left(C_{Y} \mathcal{Z}\right)_{y}$ de $C_{Y} \mathcal{Z}$ en un point $y$ de $Y$ est le cône tangent $C_{y}\left(\mathcal{Z}_{y}\right)$ à la fibre $\mathcal{Z}_{y}=\mathcal{Z} \cap \pi^{-1}(y)$ de $\mathcal{Z}$ en $y$.

Condition de PSEudo-Platitude normale (ppn). — La projection

$$
p: C_{Y} \mathcal{Z} \longrightarrow Y
$$

est ouverte pour toute strate $Y$ de $\mathcal{Z}$.

Nous rappelons au $\S 2$ les conditions de régularité usuelles, dont nous aurons besoin, en particulier la condition $(a)$ de Whitney. Quand une stratification vérifie deux conditions, par exemple est $(a)$-régulière et $(n)$-régulière, nous dirons qu'elle est $(a+n)$-régulière, pour la simplicité des notations. Les stratifications sous-analytiques vérifiant les conditions $(a+n)$ ou $(p p n)$ ont un cône normal ayant un bon comportement du point de vue de la dimension des fibres. En effet, elles vérifient la condition

$$
\operatorname{dim}\left(C_{Y} \mathcal{Z}\right)_{y} \leq \operatorname{dim} \mathcal{Z}-\operatorname{dim} Y-1
$$

C'est évident pour $(a+n)$, et pour $(p p n)$ cela résulte de $\left(5.1 \mathrm{ii}^{\prime}\right)$ (voir aussi [4], [5, lemme 2.4]). Pour des stratifications différentiables il y a le problème de savoir ce que c'est que la dimension.

Malgré cette limitation, le cône tangent $C_{y}\left(\mathcal{Z}_{y}\right)$ à la fibre $\mathcal{Z}_{y}=\mathcal{Z} \cap \pi^{-1}(y)$ (et donc la fibre $\left(C_{Y} \mathcal{Z}\right)_{y}$ du cône normal, supposant $\left.(n)\right)$ peut être assez arbitraire : des travaux récents de Ferrarotti, Fortuna et Wilson montrent que tout cône semi-algébrique fermé de codimension $\geq 1$ est réalisé comme le cône tangent en un point d'une certaine variété algébrique réelle [6], et Kwiecinski et Trotman ont montré que tout cône fermé est réalisé comme le cône tangent en une singularité isolée d'un certain espace stratifié $C^{\infty}(b)$-régulier [14].

TOME $130-2002-\mathrm{N}^{\mathrm{O}} 1$ 
Les premiers résultats dans la direction de notre étude ont été obtenus par Hironaka, qui a montré dans [10] qu'une stratification de Whitney (i.e. (b)-régulière) d'un ensemble analytique (réel ou complexe) est normalement pseudoplate le long de chaque strate. J.-P. Henry et M. Merle [9] ont montré l'assertion analogue à $(n)$ avec $\mathcal{Z}$ remplacé par $X \cup Y$ quand $X$ et $Y$ sont deux strates adjacentes d'une stratification de Whitney sous-analytique de $X \cup Y$. Un exemple algébrique réel de [2] montre qu'il ne suffit pas en général que la stratification soit $(a+\delta)$-régulière.

Dans [17], nous avons étendu le résultat de Henry et Merle au cadre différentiable, avec l'hypothèse que la stratification vérifie les conditions $(w)$ de Verdier et $(\delta)$ de Bekka-Trotman. Ici nous améliorons les résultats de [17] par un affaiblissement de la régularité imposée à la stratification. Nous montrons dans le théorème 3.1 que $(n)$ est vérifiée par toute stratification différentiable (a)-régulière ayant en plus une régularité $\left(r^{e}\right)$, que nous introduisons ici.

Toute stratification $C^{2}(w)$-régulière vérifie automatiquement $(a)$ et $\left(r^{e}\right)$, c'est-à-dire $\left(a+r^{e}\right)$. Pour des strates sous-analytiques la combinaison $\left(a+r^{e}\right)$ est équivalente (proposition 2.5) au critère $(r)$ introduit par T.-C. Kuo en 1971, ce qui entraîne la condition (b) de Whitney [13]; on sait depuis [19] que $(r)$ est strictement plus faible que $(w)$ dans le cas semi-algébrique, et il existe même des exemples algébriques réels [3]. L'équivalence de $(b),(r)$ et $(w)$ pour les stratifications analytiques complexes est connue depuis 1982 ([18], [8]).

Nous montrons aussi, dans la proposition 5.2, la pseudo-platitude normale de toute stratification $\left(a+r^{e}\right)$-régulière. L'exemple 4.2 (un « escargot de Kuo », déjà utilisé par nous dans [16]) montre qu'une stratification différentiable $(b)$ régulière ne vérifie pas forcément $(n)$ ou $(p p n)$. À la fin du $\$ 5$ nous décrivons des exemples algébriques montrant qu'il n'y a pas d'implication entre les conditions $(n)$ et $(p p n)$, même en supposant la condition $(a)$.

\section{Définitions et résultats préliminaires}

Nous rappelons d'abord les définitions des conditions $(a)$ et $(b)$ de Whitney, $(r)$ de Kuo [13], $(w)$ de Kuo-Verdier [21] et $(\delta)$ de Bekka-Trotman [1], [2].

Soient $X$ et $Y$ deux sous-variétés de $\mathbb{R}^{n}$ telles que $Y \cap \bar{X} \neq \varnothing, \pi$ la projection locale sur $Y$. Suivant Hironaka [10], nous notons par $\alpha_{X, Y}(x)$ la distance de $T_{x} X$ à $T_{\pi(x)} Y$, qui s'exprime par

$$
\alpha_{X, Y}(x)=\max \left\{\langle\mu(u), \mu(v)\rangle: u \in N_{x} X-\{0\}, v \in T_{\pi(x)} Y\right\},
$$

et par $\beta_{X, Y}(x)$ la distance de $x \pi(x)$ à $T_{x} X$ exprimée par

$$
\beta_{X, Y}(x)=\max \left\{\langle\mu(u), \mu(x \pi(x))\rangle: u \in N_{x} X-\{0\}\right\},
$$

où $\langle$,$\rangle est le produit scalaire sur \mathbb{R}^{n}$. 
Pour $v \in \mathbb{R}^{n}$, la distance du vecteur $v$ à un plan $B$ s'écrit

$$
\eta(v, B)=\sup \left\{v \cdot n: n \in B^{\perp},\|n\|=1\right\} .
$$

Posons

$$
d(A, B)=\sup \{\eta(v, B): v \in A,\|v\|=1\} .
$$

Posons encore

$$
R_{X, Y}(x)=\frac{\|x\| \alpha_{X, Y}(x)}{\|x \pi(x)\|} \text { et } \quad W_{X, Y}(x, y)=\frac{d\left(T_{x} X, T_{y} Y\right)}{\|x y\|} .
$$

Lorsque aucune confusion ne sera possible, nous omettrons de préciser les indices $X$ et $Y$.

DÉfinition 2.1. - Le couple de strates $(X, Y)$ vérifie en $0 \in Y$ :

- la condition (a) si, pour $x$ dans $X$,

$$
\lim _{x \rightarrow 0} \alpha_{X, Y}(x)=0
$$

- la condition (b) si, pour $x$ dans $X$,

$$
\lim _{x \rightarrow 0} \alpha_{X, Y}(x)=\lim _{x \rightarrow 0} \beta_{X, Y}(x)=0 ;
$$

- la condition $(r)$ si, pour $x$ dans $X$,

$$
\lim _{x \rightarrow 0} R_{X, Y}(x)=0 ;
$$

- la condition $(w)$ si, pour $x$ dans $X$ et $y$ dans $Y, W_{X, Y}(x, y)$ est borné près de 0 ;

- la condition $(\delta)$ si, pour $x$ dans $X$ et $y$ dans $Y$, l'angle entre la droite $x y$ et $T_{x} X$ est borné, près de 0 , par une constante $\delta<\frac{1}{2} \pi$.

Dans cet article nous introduisons la condition $\left(r^{e}\right)$ suivante, de type KuoVerdier.

DÉfinition 2.2. - Soit $e \in[0,1[$. Nous dirons que $(X, Y)$ vérifie la condition $\left(r^{e}\right)$ en $y \in Y$ si, pour $x \in X$, la quantité suivante est bornée près de 0 :

$$
R_{e}(x)=\frac{\|y \pi(x)\|^{e} \alpha_{X, Y}(x)}{\|x \pi(x)\|} .
$$

Nous utiliserons le plus souvent $y=0$ auquel cas $R_{e}$ devient

$$
\frac{\|\pi(x)\|^{e} \alpha_{X, Y}(x)}{\|x \pi(x)\|} .
$$

Cette condition est invariante par difféomorphisme de classe $C^{2}$. Ce n'est autre que $(w)$ quand $e=0$, ainsi $(w)$ implique $\left(r^{e}\right)$ pour tout $e \in[0,1[$. Mais, contrairement à $(w)$, la condition $\left(r^{e}\right)$ quand $e>0$ n'implique pas la condition $(a)$ : on construit facilement un contre-exemple d'une surface semi-algébrique dans $\mathbb{R}^{3}$ obtenue en pinçant un demi-plan $\{z \geq 0, x=0\}$, de bord l'axe $0 y=Y$, dans une région cuspidale $\Gamma=\left\{x^{2}+y^{2} \leq z^{p}\right\}$, où $p$ est un entier impair tel

TOME $130-2002-\mathrm{N}^{\mathrm{O}} 1$ 
que $p>2 / e$, de telle façon que dans $\Gamma$ il y ait des suites tendant vers 0 pour lesquelles la condition $(a)$ ne soit pas vérifiée. On peut vérifier que cet exemple est $\left(r^{e}\right)$-régulier.

Il est bien souvent utile de savoir que l'intersection transverse de deux stratifications régulières est encore régulière, et nous aurons besoin de cette propriété pour la condition $\left(a+r^{e}\right)$ dans la preuve du théorème 3.1, dans le cas où l'une des stratifications est une seule variété lisse. Rappelons tout d'abord la notion d'intersection transverse de deux stratifications :

DÉFInition 2.3. - Soient $\Sigma$ et $\Sigma^{\prime}$ deux stratifications. Nous dirons qu'elles sont transverses si pour toutes strates $X$ de $\Sigma$ et $X^{\prime}$ de $\Sigma^{\prime}$ les variétés $X$ et $X^{\prime}$ sont transverses. La stratification intersection $\Sigma \cap \Sigma^{\prime}$ est celle donnée par les $X \cap X^{\prime}$ avec $X \in \Sigma$ et $X^{\prime} \in \Sigma^{\prime}$.

Une démonstration du fait que la condition $(b)$ est préservée par intersection transverse était donnée par Gibson [7], pour la condition $(a+\delta)$ voir [1] ou [2]. Pour la condition $(w)$ nous ne connaissons aucune référence : la propriété d'invariance ne semble pas avoir été énoncée sauf dans le cas d'une section par une variété lisse [21]. La démonstration du théorème suivant s'applique à toutes les conditions envisagées ci-dessus.

THÉORÈme 2.4. - Les conditions $(a),(b),(r),(w),(a+\delta)$ et $\left(a+r^{e}\right)$ pour $0 \leq e<1$ sont invariantes par intersection transverse de deux stratifications de classe $C^{2}$.

Démonstration. - Considérons tout d'abord deux plans $A$ et $B$ transverses. Pour $v \in \mathbb{R}^{n}$, la distance du vecteur $v$ à $B$ s'écrit

$$
\eta(v, B)=\sup \left\{v \cdot n: n \in B^{\perp},\|n\|=1\right\} .
$$

La distance de $v$ à $A \cap B$ s'écrit donc

$$
\eta(v, A \cap B)=\sup \left\{v \cdot n: n \in A^{\perp}+B^{\perp},\|n\|=1\right\} .
$$

Décomposons $A^{\perp}+B^{\perp}$ en $I+U+V$ où $I=A^{\perp} \cap B^{\perp}$, et $U$ (resp. $V$ ) est le complémentaire orthogonal de $I$ dans $A^{\perp}$ (resp. $B^{\perp}$ ). Alors

$$
\eta(v, A \cap B)=\sup \left\{v \cdot\left(\sum_{i=1}^{3} n_{i}\right): n_{1} \in I, n_{2} \in U, n_{3} \in V,\left\|\sum_{i=1}^{3} n_{i}\right\|=1\right\} .
$$

Soient maintenant $\Sigma$ et $\Sigma^{\prime}$ deux stratifications transverses.

Prenons deux strates $X \cap X^{\prime}$ et $Y \cap Y^{\prime}$ de $\Sigma \cap \Sigma^{\prime}$, telles que $0 \in Y \cap Y^{\prime} \cap$ $\overline{\left(X \cap X^{\prime}\right)}$, avec éventuellement $X=Y$ ou $X^{\prime}=Y^{\prime}$. Nécessairement $0 \in Y \cap \bar{X}$ et $0 \in Y^{\prime} \cap \bar{X}^{\prime}$.

Soient $U$ un voisinage de 0 et $K_{X, Y}>0$ une constante réelle, tels que

$$
d\left(T_{x} X, T_{y} Y\right) \leq K_{X, Y} \phi_{X, Y}(x, y),
$$

BULLETIN DE LA SOCIÉtÉ MATHÉMATIQUE DE FRANCE 
pour tout $x \in U^{\prime} \cap X$ et tout $y \in U \cap Y, \phi_{X, Y}$ étant une fonction donnée associée à la condition de régularité étudiée, et soient $U^{\prime}$ un voisinage de 0 et $K_{X^{\prime}, Y^{\prime}}>0$ une constante réelle, tels que

$$
d\left(T_{x} X^{\prime}, T_{y} Y^{\prime}\right) \leq K_{X^{\prime}, Y^{\prime}} \phi_{X^{\prime}, Y^{\prime}}(x, y),
$$

pour tout $x \in U^{\prime} \cap X^{\prime}$ et tout $y \in U^{\prime} \cap Y^{\prime}$.

Chaque cas se déduit de la preuve que nous donnons maintenant pour $\left(a+r^{e}\right)$. Pour cette condition, la fonction est

$$
\phi_{X, Y}(x, y)=\frac{\|x y\|}{\left\|\pi_{Y}(x)\right\|^{e}},
$$

où $\pi_{Y}$ est la projection locale sur $Y$. Ainsi

$$
\phi_{X^{\prime}, Y^{\prime}}(x, y)=\frac{\|x y\|}{\left\|\pi_{Y^{\prime}}(x)\right\|^{e}} .
$$

Nous utilisons ici le fait que $Y$ et $Y^{\prime}$ sont de classe $C^{2}$, pour que $\pi_{Y}$ et $\pi_{Y^{\prime}}$ soient définies [11].

Soit $U_{1}$ un voisinage de 0 tel que $\left\|\pi_{Y \cap Y^{\prime}}(x)\right\| \leq 2\left\|\pi_{Y}(x)\right\|$ si $x \in U_{1}$, et soit $U_{2}$ un voisinage de 0 tel que $\left\|\pi_{Y \cap Y^{\prime}}(x)\right\| \leq 2\left\|\pi_{Y^{\prime}}(x)\right\|$ si $x \in U_{2}$ (nous pourrions remplacer 2 par une constante $k>1$ quelconque, mais nous garderons 2 pour simplifier les notations).

Alors, comme sur $U_{1}$

$$
\frac{\|x y\|}{\left\|\pi_{Y}(x)\right\|^{e}} \leq \frac{2^{e}\|x y\|}{\left\|\pi_{Y \cap Y^{\prime}}(x)\right\|^{e}}
$$

on obtient l'inégalité

$$
\phi_{X, Y}(x, y) \leq 2 \phi_{X \cap X^{\prime}, Y \cap Y^{\prime}}(x, y)
$$

si $x \in X \cap U \cap U_{1}$ et $y \in Y \cap U \cap U_{1}$, parce que $0 \leq e<1$.

De même, si $x \in X^{\prime} \cap U^{\prime} \cap U_{2}$ et $y \in Y^{\prime} \cap U^{\prime} \cap U_{2}$,

$$
\phi_{X^{\prime}, Y^{\prime}}(x, y) \leq 2 \phi_{X \cap X^{\prime}, Y \cap Y^{\prime}}(x, y) .
$$

En utilisant ce qui précède pour $A=T_{x} X$ et $B=T_{x} X^{\prime}$, si $v \in T_{y} Y \cap T_{y} Y^{\prime}$ et $\|v\|=1$, en notant

$$
\left.J=\left\{\left(n_{1}, n_{2}, n_{3}\right) \in I \times U \times V, \text { telle que }\left\|\sum_{i=1}^{3} n_{i}\right\|=1\right)\right\},
$$

TOME $130-2002-\mathrm{N}^{\mathrm{O}} 1$ 
nous avons que,

$$
\begin{aligned}
\eta(v, A \cap B) & =\sup \left\{v \cdot\left(\sum_{i=1}^{3} n_{i}\right):\left(n_{1}, n_{2}, n_{3}\right) \in J\right\} \\
& \leq \sup \left\{\left(\sum_{i=1}^{3}\left|v \cdot n_{i}\right|\right):\left(n_{1}, n_{2}, n_{3}\right) \in J\right\} \\
& \leq \sup \left\{\left(\sum_{i=1}^{2}\left\|n_{i}\right\|\right) K_{X Y} \phi_{X Y}(x, y)+\left\|n_{3}\right\| K_{X^{\prime} Y^{\prime}} \phi_{X^{\prime} Y^{\prime}}:\right. \\
& \left.\quad\left(n_{1}, n_{2}, n_{3}\right) \in J\right\} \\
& \leq \sup \left\{\left(\sum_{i=1}^{3}\left\|n_{i}\right\|\right):\left(n_{1}, n_{2}, n_{3}\right) \in J\right\} K \phi(x, y),
\end{aligned}
$$

où $K=2 \max \left(K_{X Y}, K_{X^{\prime} Y^{\prime}}\right)$ et $\phi(x, y)=\phi_{X \cap X^{\prime}, Y \cap Y^{\prime}}(x, y)$. Comme

$$
\left\|\sum_{i=1}^{3} n_{i}\right\|^{2}=\left\|n_{1}\right\|^{2}+\left\|n_{2}\right\|^{2}+\left\|n_{3}\right\|^{2}+2 \cos \left(n_{2}, n_{3}\right)\left\|n_{2}\right\| \cdot\left\|n_{3}\right\|=1
$$

et $\left\{\sum_{i=1}^{3}\left\|x_{i}\right\|^{2}+2 a\left\|x_{2}\right\| \cdot\left\|x_{3}\right\|=1\right\}$ est compact pour $\|a\| \neq 1$, nous avons que

$$
\begin{aligned}
d\left(T_{y} Y \cap T_{y} Y^{\prime}, A \cap B\right) & =\sup \left\{\eta(v, A \cap B): v \in T_{y} Y \cap T_{y} Y^{\prime},\|v\|=1\right\} \\
& \leq C K \phi(x, y),
\end{aligned}
$$

où

$$
C=\sup \left\{\left(\sum_{i=1}^{3}\left\|x_{i}\right\|\right): \sum_{i=1}^{3}\left\|x_{i}\right\|^{2}+2 a\left\|x_{2}\right\| \cdot\left\|x_{3}\right\|=1,\|a\|<1-\epsilon\right\},
$$

et $\epsilon$ est donné par l'angle minimal de $T_{x} X$ et $T_{x^{\prime}} X^{\prime}$ sur un voisinage de $y ; \epsilon$ étant non-nul par la $(a)$-régularité de $(X, Y)$ et de $\left(X^{\prime}, Y^{\prime}\right)$ en $y$, et la transversalité de $Y$ et $Y^{\prime}$ en $y$. Nous laissons au lecteur le soin de vérifier que $(a)$ est satisfaite pour $\Sigma \cap \Sigma^{\prime}$, en utilisant l'inégalité $\left(^{*}\right)$. Le théorème en résulte puisque $\phi(x, y)=\phi_{X \cap X^{\prime}, Y \cap Y^{\prime}}(x, y)$.

LEMme 2.5. - Si $(a)$ est vérifiée, et $\left(r^{e}\right)$ a lieu pour un certain e $\in[0,1[$, alors $(r)$ est vérifiée.

Démonstration. - De $\left(r^{e}\right)$ il résulte que

$$
\frac{\|\pi(x)\| \alpha(x)}{\|x \pi(x)\|} \leq C\|\pi(x)\|^{1-e}
$$

et donc que

$$
R(x)=\frac{\|\pi(x)\| \alpha(x)}{\|x \pi(x)\|} \frac{\|x\|}{\|\pi(x)\|}
$$

BULLETIN DE LA SOCIÉTÉ MATHÉMATIQUe DE FRANCE 
tend vers zéro parce que $e<1$, sauf éventuellement si $\|\pi(x)\| /\|x\|$ tend vers zéro. Mais dans ce cas $(r)$ résulte de la condition $(a)$.

Proposition 2.6. - Pour des strates sous-analytiques, $(r)$ implique $(a)$ et l'existence de $e \in] 0,1\left[\right.$ tel que $\left(r^{e}\right)$ soit vérifiée. Ainsi $(r)$ devient équivalente $\grave{a}\left(a+r^{e}\right)$ pour un certain $e, 0<e<1$.

Démonstration. - Supposons que $R(x)$ tend vers zéro. On constate déjà que (a) est vérifiée, parce que

$$
\frac{\|x\|}{\|x \pi(x)\|} \geq 1 .
$$

Par inégalité de Lojasiewicz, il existe $\lambda>0$ tel que $R(x) \leq C\|x\|^{\lambda}$ et on peut évidemment supposer que $0<\lambda<1$. Puisque $\|\pi(x)\| /\|x\|$ est borné par 1 , on déduit facilement que

$$
R_{1-\lambda}(x)=\frac{\|\pi(x)\|^{1-\lambda} R(x)}{\|x\|^{1-\lambda}\|x\|^{\lambda}} \leq C
$$

et donc $\left(r^{e}\right)$ est vérifiée, avec $e=1-\lambda$.

Remarque 2.7. - Clairement, $\left(r^{e}\right)$ implique que $R_{f}(x)$ tend vers 0 pour tout $e<f<1$, et aussi que $\|\pi(x)\| \alpha(x) /\|x \pi(x)\|$ tend vers 0 . Dans le cas sousanalytique il est facile de voir, utilisant une inégalité de Łojasiewicz, que $\left(r^{e}\right)$ est équivalente à ce que $R_{f}(x)$ tend vers 0 pour un certain $e<f<1$.

\section{La fibre du cône normal}

Dans cette section nous démontrons le résultat suivant :

ThÉorème 3.1. - Soit $\mathcal{Z}$ un fermé, stratifié par des variétés de classe $C^{k}$, $k \geq 2$, de manière $\left(a+r^{e}\right)$-régulière relativement à une strate $Y$. Alors $C_{y}\left(\mathcal{Z}_{y}\right)=\left(C_{Y} \mathcal{Z}\right)_{y}$, pour tout point y de $Y$, c'est-à-dire que $(n)$ est vérifiée.

On déduit immédiatement le corollaire suivant, et on déduit aussi le théorème de [9], que $(b)$ implique $(n)$ pour des stratifications sous-analytiques, en remarquant que $(r)$ est équivalente à $(b)$ en dimension 1 pour des strates sousanalytiques [13], et en appliquant la proposition 2.5 ci-dessus ( $c f$. . [9], [17]).

Corollaire 3.2. - Si $\mathcal{Z}$ possède une stratification $\left(a+r^{e}\right)$-régulière de classe $C^{k}, k \geq 2$, alors $C_{y}\left(\mathcal{Z}_{y}\right)=\left(C_{Y} \mathcal{Z}\right)_{y}$, pour toute strate $Y$ et pour tout point $y$ de $Y$.

Le corollaire 3.2 améliore le théorème 2.1 de [17], où nous avons obtenu la même conclusion utilisant une hypothèse plus forte : que la stratification soit $(w+\delta)$-régulière. Rappelons que $(w)$ entraine $(a)$ et $\left(r^{e}\right)$ quel que soit $e \geq 0$.

TOME $130-2002-\mathrm{N}^{\mathrm{O}} 1$ 
Démonstration du théorème. - L'inclusion $C_{y}\left(\mathcal{Z}_{y}\right) \subset\left(C_{Y} \mathcal{Z}\right)_{y}$ est évidente. Soit $v$ un élément de $\left(C_{Y} \mathcal{Z}\right)_{y}$. Il existe une suite de points $x_{i} \in \mathcal{Z}$ telle que la limite de $\mu\left(x_{i} \pi\left(x_{i}\right)\right)$ soit égale à $v$. Soit $y_{i}$ le point $\pi\left(x_{i}\right)$. On peut supposer, quitte à extraire une sous-suite, que les $y_{i}$ sont tracés sur $\gamma, \operatorname{arc} C^{1}$ et $C^{k}$ en dehors de $y$ (par le théorème d'extension $C^{1}$ de Whitney comme dans [20]). Les tangentes à $\gamma$ déterminent un champ de vecteurs $C^{k-1}$ unité, noté $\delta$. Désignons par $\mathcal{Z}_{\gamma}$ l'ensemble $\mathcal{Z} \cap \pi^{-1}(\gamma)$ — intersection transverse de $\mathcal{Z}$ et de la variété lisse $\pi^{-1}(\gamma)$ - et par $p_{x}$ la projection sur l'espace tangent à la strate de $\mathcal{Z}_{\gamma}$ passant par $x$. Nous noterons $\kappa$ la restriction de $\pi$ à $\mathcal{Z}_{\gamma}$. Posant

$$
w_{\mathcal{Z}}(x)=p_{x}\left(\delta_{\kappa(x)}\right) \in T_{x} \mathcal{Z}_{\gamma},
$$

en particulier $w_{\mathcal{Z}}(x)=\delta_{x}$ pour $x$ dans $Y$, on définit un champ de vecteurs $C^{k-1}$ sur les strates de $\mathcal{Z}_{\gamma}-\kappa^{-1}(y)$, vérifiant

$$
\left\|w_{\mathcal{Z}}(x)-w_{\mathcal{Z}}(\kappa(x))\right\| \leq C \frac{\|x \kappa(x)\|}{\|\kappa(x)\|^{e}}
$$

par invariance de $\left(a+r^{e}\right)$ par intersection transverse - grâce au théorème 2.4 qui est valide pour $\mathcal{Z} \cap \pi^{-1}(\gamma)$ bien que la classe de $\pi^{-1}(\gamma)$ soit $C^{1}$ en 0 , la projection étant bien définie comme restriction de $\pi$. Par la $(a)$-régularité, $w_{\mathcal{Z}}$ et $\kappa_{*}\left(w_{\mathcal{Z}}\right)$ ne s'annulent pas sur un voisinage de $y$. En posant

$$
v_{\mathcal{Z}}=\frac{w_{\mathcal{Z}}}{\left\|\kappa_{*}\left(w_{\mathcal{Z}}\right)\right\|}
$$

on obtient un champ vérifiant encore $\left({ }^{* *}\right)$ et $\kappa_{*}\left(v_{\mathcal{Z}}\right)=\delta$. Ce champ de vecteurs n'est peut-être pas continu, mais il est intégrable sur chaque strate au-dessus de $\gamma-\{y\}:$

LEMME 3.3. - Chaque courbe intégrale du champ $v_{\mathcal{Z}}$ atteint $\pi^{-1}(y)=\kappa^{-1}(y)$ sans arriver à $y$.

Démonstration. - Soit $\phi$ une courbe intégrale de $v_{\mathcal{Z}}$. Notons tout d'abord que le rapport $\kappa(\phi(s)) / s$ est borné, ce qui nous permet, quitte à modifier certaines constantes, de remplacer $\kappa(\phi(s))$ par $s$ dans les calculs qui suivent. La longueur d'une courbe intégrale de $w_{\mathcal{Z}}$, sur un intervalle $[a, b]$, est majorée par

$$
\int_{a}^{b}\left(1+C\|\kappa(\phi(s))\|^{-e}\right) \mathrm{d} s
$$

puisque

$$
\left\|\delta-v_{\mathcal{Z}}\right\| \leq C\|\phi \kappa(\phi)\| \cdot\|\kappa(\phi)\|^{-e}
$$

et donc, est aussi majorée par $(b-a)+C^{\prime}\left(b^{1-e}-a^{1-e}\right)$. Ceci montre que les courbes intégrales atteignent $\kappa^{-1}(y)$. Pour montrer qu'elles n'atteignent pas $y$, posons

$$
f(t)=\|\phi(t) \kappa(\phi(t))\| .
$$

BULletin DE LA SOCiÉtÉ MATHÉmATiQUe DE FRANCE 
Alors

Comme

$$
f^{\prime}=\frac{\left(\kappa\left(\phi^{\prime}\right)-\phi^{\prime}, \phi \kappa(\phi)\right)}{\|\phi \kappa(\phi)\|}
$$

$$
\left\|\kappa\left(\phi^{\prime}\right)-\phi^{\prime}\right\|=\left\|\delta-v_{\mathcal{Z}}\right\| \leq C\|\phi \kappa(\phi)\| \cdot\|\kappa(\phi)\|^{-e}
$$

on obtient que $\left|f^{\prime} / f\right| \leq C\|\kappa(\phi)\|^{-e}$ et, sur un intervalle $[a, b]$,

$$
f(t) \geq \exp \left(-C^{\prime} b^{1-e}+\log (f(a))\right) .
$$

Puisque $\phi$ atteint $\kappa^{-1}(y)$ en un temps fini on a le résultat.

LEMME 3.4. - Le mouvement latéral des sécantes tend vers 0.

Démonstration. - La valeur absolue de la variation $\Delta\left(t_{1}, t_{2}\right)$ de la sécante sur l'intervalle de temps $\left[t_{1}, t_{2}\right]$ est

$$
\left\|\Delta\left(t_{1}, t_{2}\right)\right\|=\left|\int_{t_{1}}^{t_{2}} \frac{\mathrm{d}}{\mathrm{d} s}(\mu(\phi(s) \kappa(\phi(s)))) \mathrm{d} s\right| \leq \int_{t_{1}}^{t_{2}} 2 \frac{\left\|\kappa\left(\phi^{\prime}\right)-\phi^{\prime}\right\|}{\|\phi \kappa(\phi)\|} .
$$

Dans le lemme précédent on a vu que $\left\|\kappa\left(\phi^{\prime}\right)-\phi^{\prime}\right\| \leq C\|\phi \kappa(\phi)\| \cdot\|\kappa(\phi)\|^{-e}$, et donc

$$
\left\|\Delta\left(t_{1}, t_{2}\right)\right\| \leq 2 C \int_{t_{1}}^{t_{2}} \frac{\mathrm{d} s}{s^{e}}=2 C\left(t_{2}^{1-e}-t_{1}^{1-e}\right)
$$

qui tend vers 0 avec $t_{1}, t_{2}$.

Fin de la démonstration du théorème 3.1. - Désignant par $z_{i}$ le point d'intersection de la courbe intégrale passant par $x_{i}$ avec $\kappa^{-1}(y)$,

$$
\left\|\mu\left(z_{i} \kappa\left(z_{i}\right)\right)-v\right\| \leq\left\|\mu\left(z_{i} \kappa\left(z_{i}\right)\right)-\mu\left(x_{i} \kappa\left(x_{i}\right)\right)\right\|+\left\|\mu\left(x_{i} \kappa\left(x_{i}\right)\right)-v\right\| .
$$

Chacune des quantités $\left\|\mu\left(z_{i} \kappa\left(z_{i}\right)\right)-\mu\left(x_{i} \kappa\left(x_{i}\right)\right)\right\|$ et $\left\|\mu\left(x_{i} \kappa\left(x_{i}\right)\right)-v\right\|$ tend vers zéro d'après ce qui précède, d'où le théorème.

\section{Exemples et remarques}

Remarque 4.1. - La condition $\left(a+r^{e}\right)$ n'est pas nécessaire pour avoir la trivialité du cône normal. Pour un produit direct $A \times \mathbb{R}$, le cône normal est trivial le long de $\mathbb{R}$, sans que $A$ ait de régularité particulière. Pire, il est possible que $A$ vérifie $\left(a+r^{e}\right)$ sans que $A \times \mathbb{R}$ la vérifie [3] !

Exemple 4.2. - Un escargot de Kuo, cf. [16]. Soit $\mathcal{Z} \subset \mathbb{R}^{3}$ le sous-ensemble suivant :

$$
\mathcal{Z}=\left\{(r, \theta, z): r=e^{-\theta^{2} / z^{3}} \text { pour } z>0, y=0, x \in[0,1[\text { pour } z \leq 0\} .\right.
$$

La stratification donnée par $Y=(0 z)$ et $X=\mathcal{Z}-Y$ est $(b)$-régulière, et donc vérifie $(\delta)$, pour tout $\delta>0$. Le long de l'axe des $z$, le cône normal est $S^{1}$ pour $z>0$, un point pour $z \leq 0$. Ainsi le résultat du théorème 3.1 n'est pas valable dans ce cas. Bien entendu l'exemple n'est pas sous-analytique. On peut noter

TOME $130-2002-\mathrm{N}^{\mathrm{O}} 1$ 
aussi que le rapport $d\left(T_{x} X, Y\right) / d(x, Y)$ est de l'ordre de $1 / z^{4}$ ce qui montre que $\left(r^{e}\right)$ n'est vérifiée en 0 pour aucun $e$ de $[0,1[$. On constate enfin que la projection du cône normal de $\mathcal{Z}$ le long de $Y$ n'est pas ouverte au-dessus de 0 .

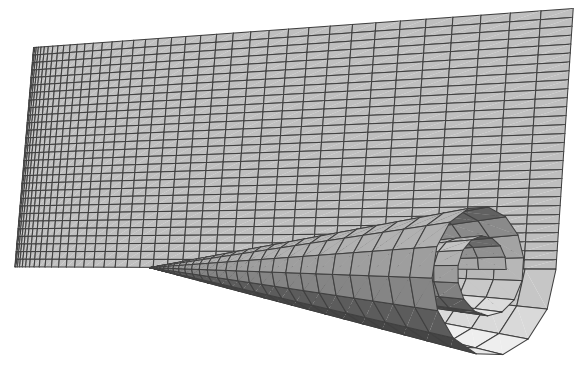

Figure 1. Un escargot de Kuo

EXEMPLE 4.3. - Soient $m$ et $r$ deux réels strictement positif. Un tumulus (voir [12], [15]) de paramètres $m$ et $r$ centré en 0 , est la surface de $\mathbb{R}^{3}$, notée $T_{m, r}$ et donnée par

$$
\left\{\left(x_{1}, x_{2}, x_{3}\right): m^{7} r^{3} x_{3}=\left(x_{1}^{2}-m^{2} r^{2}\right)^{2}\left(x_{2}^{2}-m^{2}\right)^{2},\left|x_{1}\right| \leq m r,\left|x_{2}\right| \leq m\right\} .
$$

Une propriété remarquable de ces tumuli est que lorsque $m, r$ tendent vers 0 , la structure normale tend vers

$$
\left\{\left(-4 \xi\left(\xi^{2}-1\right)\left(\xi^{2}-1\right)^{2}, 0,1\right): \xi \in[-1,1]\right\} .
$$

Si l'on affaiblit $(w)$ en $\left(w_{\beta}\right), \beta<1$, c'est-à-dire si on suppose que le rapport $d\left(T_{x} X, T_{z} Y\right) /\|x \kappa(x)\|^{\beta}$ est borné près de $y$ pour $x$ dans $X$ et $z$ dans $Y$, alors le résultat du corollaire 3.2 n'est pas conservé.

Considérons pour cela le demi-plan $x_{3}=0, x_{1}>0$ dans $\mathbb{R}^{3}$, et notons $C_{\alpha}$ le morceau de courbe $\left\{x_{1}=x_{2}^{2+\alpha / \alpha}, x_{1}>0\right\}$, qui est tangent à $\left(0 x_{2}\right)$. Centrons aux points $\left(x_{1}^{i}, x_{2}^{i}, 0\right)=\left(r_{i}^{1+\alpha}, r_{i}^{\alpha(1+\alpha) /(2+\alpha)}, 0\right)$ des tumuli $T_{r_{i}^{\alpha}, r_{i}}$, avec une suite $r_{i}$ qui tend vers 0 de sorte que les tumuli soient disjoints.

Alors, si l'on note $X$ le demi-plan perturbé le long de $C_{\alpha}$ et $Y=\left(0 x_{2}\right)$, on obtient une stratification $\left(w_{1 /(1+\alpha)}\right)$-régulière, pour laquelle le cône normal n'est pas obtenu dans la fibre. En effet, en notant $\pi$ la projection sur $Y$, et en notant

sur les tumuli, nous avons que

$$
\xi=\frac{x_{1}-x_{1}^{i}}{r^{1+\alpha}} \quad \text { et } \quad \chi=\frac{x_{2}-x_{2}^{i}}{r^{\alpha}}
$$

$$
\|x-\pi(x)\| \equiv \frac{3}{2} r^{1+\alpha}, \quad \text { et } \quad d\left(T_{x} X, Y\right) \equiv-4 \chi\left(\chi^{2}-1\right)\left(\xi^{2}-1\right)^{2} r
$$

BULLETIN DE LA SOCIÉTÉ MATHÉMATIQUE DE FRANCE 
de sorte que

$$
\frac{d\left(T_{x} X, Y\right)}{\|x-\pi(x)\|^{\beta}} \leq \text { Cte, } \quad \text { avec } \quad \beta=\frac{1}{1+\alpha},
$$

c'est-à-dire que la stratification obtenue est $\left(w_{\beta}\right)$-régulière. De plus les fibres du cône tangent le long de $Y$ sont des points, sauf en 0 où l'on a une courbe, étant donné que l'angle des sécantes passant par le sommet des tumuli a une ouverture constante (la tangente de cet angle est $\frac{2}{3}$ ). Il est clair par la construction que les limites des sécantes en 0 ne sont pas obtenues dans la fibre de $\pi$, c'est-à-dire que la condition $(n)$ n'est pas vérifiée.

Dans les deux exemples précédents, les strates telles qu'elles sont données sont de classe $C^{1}$, mais elles peuvent être lissées sans difficulté de manière à obtenir des stratifications $C^{2}$ ayant les mêmes propriétés.

\section{Ouverture de la projection}

Ce qui précède permet de montrer aussi l'ouverture de la projection du cône normal sur $Y$. Pour voir cela montrons tout d'abord le résultat suivant $(\overline{\mathbb{N}}=\mathbb{N} \cup \infty$ avec sa topologie usuelle).

Lemme 5.1. - Soient $M$ et $Y$ deux variétés différentielles, $\mathcal{Z}$ un sousensemble de $M$ et $f$ une application continue de $M$ dans $Y$. Les assertions suivantes sont équivalentes :

(i) $f: \mathcal{Z} \rightarrow Y$ est ouverte;

(ii) pour tout chemin continu $h: \overline{\mathbb{N}} \rightarrow Y$, le produit fibré $\mathcal{Z}_{h}=\overline{\mathbb{N}} \times_{h} \mathcal{Z}$ est égal à l'adhérence de $f_{h}^{-1}(\overline{\mathbb{N}}-\{\infty\})$, où $f_{h}$ est l'image réciproque de $f$ par $h$; et dans le cas sous-analytique $(M, Y$ analytiques, $f, \mathcal{Z}$ sous-analytiques, $f$ propre)

(ii') pour tout chemin analytique $h: \mathbb{R} \rightarrow Y$, le produit fibré $\mathcal{Z}_{h}=\mathbb{R} \times_{h} \mathcal{Z}$ est égal à l'adhérence de $f_{h}^{-1}(\mathbb{R}-\{0\})$.

Démonstration. - Montrons que (i) implique (ii). Supposons $f$ ouverte. Soit $\overline{\mathbb{N}} \stackrel{h}{\longrightarrow} Y$ une suite dans $Y$. Si (ii) n'est pas satisfaite, alors il existe $(\infty, z)$ dans $\mathcal{Z}_{h}-\overline{f_{h}^{-1}(\overline{\mathbb{N}}-\{\infty\})}$, et un voisinage ouvert $V$ de $(\infty, z)$, de la forme ]$n, \infty] \times V_{1}$, tel que $V \cap \overline{f_{h}^{-1}(\overline{\mathbb{N}}-\{\infty\})}$ soit vide. Comme $f$ est ouverte, $f\left(V_{1}\right)$ est un voisinage de $f(z)=h(\infty)$ et rencontre $h(\mathbb{N})$, ce qui signifie que $V$ rencontre $f_{h}^{-1}(\overline{\mathbb{N}}-\{\infty\})$, donnant une contradiction.

Montrons que (ii) implique (i). Supposons $f$ non ouverte : il existerait un ouvert $U$ de $\mathcal{Z}$ tel que $f(U)$ ne soit pas ouvert. Soit $h_{n}$ une suite de $Y \backslash f(U)$ telle que $h_{n} \rightarrow y \in f(U), f_{h}^{-1}(t)$ ne rencontre pas $\overline{\mathbb{N}} \times U$ pour $t \neq \infty$, ce qui implique que l'adhérence de $f_{h}^{-1}(\overline{\mathbb{N}}-\{0\})$ ne rencontre pas $\overline{\mathbb{N}} \times U$. Mais $y=h(\infty) \in f(U)$, et donc $(\infty, y) \in(\overline{\mathbb{N}} \times U) \cap \mathcal{Z}_{h}$, contredisant (ii).

TOME $130-2002-\mathrm{N}^{\mathrm{O}} 1$ 
Dans le cas sous-analytique, (i) implique (ii') avec la même démonstration. Pour l'implication inverse, il suffit de prendre $U$ sous-analytique. Alors $f(U)$ est sous-analytique, et on peut appliquer le lemme du chemin à son complémentaire pour trouver un chemin analytique $h: \mathbb{R} \rightarrow Y$, tel que $h(0)=y$ et $h(\mathbb{R}-\{0\}) \cap$ $f(U)=\varnothing$, et on termine de la même façon.

Proposition 5.2. - Sous les hypothèses du théorème 3.1, la projection de $C_{Y} \mathcal{Z}$ dans $Y$ est ouverte, i.e. $\mathcal{Z}$ est normalement pseudo-plate le long de $Y$.

Démonstration. - Soit $y_{n}$ une suite de points de $Y$, convergeant vers $y \in Y$. Et soit $\gamma$ un chemin de classe $C^{1}$ passant par les $y_{n}$ et $y=\gamma(0)$, de classe $C^{k}$ sur $\gamma-\{0\}$. On peut supposer que $\operatorname{Im}(\gamma)$, que l'on notera aussi $\gamma$, est une sous-variété de $Y$. Soit $v \in C_{y}\left(\mathcal{Z}_{y}\right), v=\lim _{i \rightarrow \infty}\left(\mu\left(x_{i}^{\prime} y\right)\right)$ avec $x_{i}^{\prime} \in \pi^{-1}(y)$. En suivant les courbes intégrales données par le théorème 3.1 dans l'autre sens à partir de chaque $x_{i}^{\prime}$, on construit une double suite $x_{n}^{i}$ de $\mathcal{Z}_{\gamma}$, telle que $y_{n}=\pi\left(x_{n}^{i}\right)$. Soit $v_{n}^{i}=\mu\left(x_{n}^{i} \pi\left(x_{n}^{i}\right)\right)$. En extrayant des sous-suites des $v_{n}^{i}$ successivement pour $n$ croissant, on peut supposer que pour chaque $n, v_{n}^{i}$ tende vers $c_{n} \in\left(C_{Y} \mathcal{Z}\right)_{y_{n}}=C_{y_{n}}\left(\mathcal{Z}_{y_{n}}\right)$ par le théorème 3.1. Par l'inégalité triangulaire,

$$
\left\|c_{n}-v\right\| \leq\left\|c_{n}-v_{n}^{i}\right\|+\left\|v_{n}^{i}-\mu\left(x_{i}^{\prime} y\right)\right\|+\left\|\mu\left(x_{i}^{\prime} y\right)-v\right\| .
$$

Fixons $\epsilon>0$, et soit $i_{0}$ tel que $\left\|\mu\left(x_{i_{0}}^{\prime} y\right)-v\right\| \leq \frac{1}{3} \epsilon$. La preuve du lemme 3.4 montre que

$$
\left\|v_{n}^{i}-\mu\left(x_{i}^{\prime} y\right)\right\| \leq C\left(\left\|y_{n}\right\|^{1-e}-\|y\|^{1-e}\right)
$$

uniformément en $i$, et il est alors facile de trouver $n_{0}$ tel que, pour $n \geq n_{0}$, $\left\|c_{n}-v_{n}^{i_{0}}\right\|<\frac{1}{3} \epsilon$ et $\left\|v_{n}^{i_{0}}-\mu\left(x_{i_{0}}^{\prime} y\right)\right\|<\frac{1}{3} \epsilon$. On en déduit le résultat en utilisant la caractérisation (ii) du lemme précédent.

EXEMPLE 5.3. - Reprenons l'exemple 4.3. Les stratifications obtenues sont $\left(w_{\beta}\right)$-régulières (donc $(a)$-régulières) et telles que la projection du cône normal n'est pas ouverte : comme nous l'avons vu les fibres du cône tangent le long de $Y=(0 y)$ sont des points sauf en 0 , où la fibre est de dimension 1 , et donc la projection ne peut pas être ouverte.

REMARQUE $5.4((a+n)$ n'implique pas $(p p n))$. — L'exemple précédent peut être modifié de sorte que la stratification obtenue soit $(a)$-régulière et que le cône normal soit obtenu dans la fibre en 0 de la projection sur $Y$.

En effet, centrons une suite de tumuli $T_{m_{i}, m_{i}}$ aux points $\left(m_{i}^{2}, 0\right)$ de l'axe $(0 x)$, où $m_{i} \rightarrow 0$ et les $m_{i}$ soient tels que les tumuli ne se rencontrent pas. Notons encore $X$ la surface obtenue, et $Y=(0 y)$. Les tumuli donnent naissance à un cône tangent limite en 0 de dimension 1 , provenant de suites de points situés sur l'axe $(0 x)$. Les fibres du cône tangent le long de $Y=(0 y)$ sont encore des points sauf en 0 , où la fibre est de dimension 1, et la projection n'est donc pas ouverte. La condition $(a)$ est vérifiée - il suffit de constater que les normales limites en 0 sont dans le plan $(0 x z)$. 
G. Valette et le deuxième auteur ont observé comment construire un exemple algébrique de stratification $(a+n)$-régulière ne vérifiant pas $(p p n)$. La surface donnée en coordonnées cylindriques par $\left\{r=\left(z^{2}+\sin ^{2} \theta\right) \cos \theta\right\}$, et représentée dans sa partie $z \geq 0$, a l'aspect suivant :

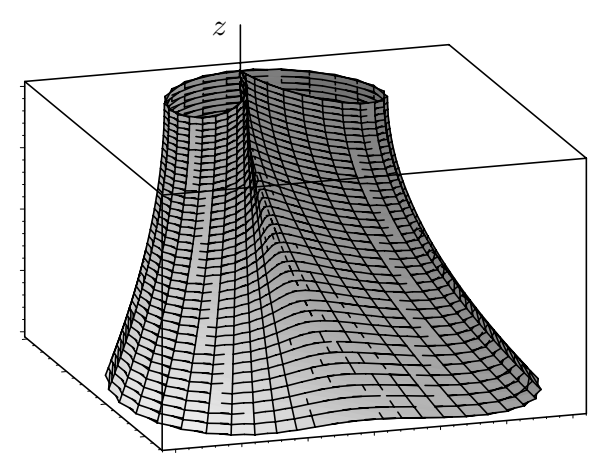

Figure 2. La surface $r=\left(z^{2}+\sin ^{2} \theta\right) \cos \theta$ (le plan $z=0$ est en haut, le plan $z=1$ est en bas)

Remarque $5.5((a+p p n)$ n'implique pas $(n))$. — G. Valette nous a communiqué l'exemple algébrique réel suivant, vérifiant $(a+p p n)$ sans vérifier $(n)$ : il suffit de poser $\mathcal{Z}=\left\{y^{2}=z^{2} x^{2}+x^{3}\right\}$ et $Y=(0 z)$ !

\section{BIBLIOGRAPHIE}

[1] BeKka (K.) - Sur les propriétés topologiques des espaces stratifiés, Thèse, Université Paris-Sud, Orsay, 1988.

[2] Bekka (K.) \& Trotman (D.) - Weakly Whitney stratified sets, in Real and Complex singularities (Bruce (J.W.) \& Tari (F.), éds.), Research Notes in Math., vol. 412, Chapman and Hall, 2000, pp. 1-15.

[3] Brodersen (H.) \& Trotman (D.) - Whitney (b)-regularity is strictly weaker than Kuo's ratio test for real algebraic stratifications, Math. Scand., t. 45 (1979), pp. 27-34.

[4] Comte (G.) - Densité et images polaires en géométrie sous-analytique, Thèse, Université de Provence, 1998.

[5] _ Équisingularité réelle : nombres de Lelong et images polaires, Ann. Sci. Éc. Norm. Sup., 4ème série, t. 33 (2000), pp. 757-788.

[6] Ferrarotti (M.), Fortuna (E.) \& Wilson (L.) - Real algebraic varieties with prescribed tangent cones, Pacific J. Math., t. 194 (2000), pp. 315323.

TOME $130-2002-\mathrm{N}^{\mathrm{O}} 1$ 
[7] Gibson (C.G.) - Construction of canonical stratifications, in Topological stability of smooth mappings, Lecture Notes in Math., vol. 552, SpringerVerlag, New York, 1976, pp. 9-34.

[8] Henry (J.-P.) \& Merle (M.) - Limites de normales, conditions de Whitney et éclatement d'Hironaka, in Singularities, Part 1 (Arcata, Calif., 1981), Proc. Sympos. Pure Math., vol.40, Amer. Math. Soc, 1983, pp. $575-584$.

[9] _ Stratifications de Whitney d'un ensemble sous-analytique, C. R. Acad. Sci. Paris, série I, t. 308 (1989), pp. 357-360.

[10] Hironaka (H.) - Normal cones in analytic Whitney stratifications, Publ. Math. I.H.E.S., t. 36 (1969), pp. 127-138.

[11] Hirsch (M. W.) - Differential Topology, Graduate Texts in Math., vol. 33, Springer-Verlag, New York, 1997.

[12] Kambouchner (A.) \& Trotman (D.) - Whitney (a)-faults which are hard to detect, Ann. Sci. Éc. Norm. Sup., 4ème série, t. 12 (1979), pp. 465471.

[13] KuO (T.-C.) - The ratio test for analytic Whitney stratifications, in Proc. of Liverpool Singularities Symposium I (Wall (C.T.C.), éd.), Lecture Notes in Math., vol. 192, Springer-Verlag, New York, 1971, pp. 141-149.

[14] Kwiecinski (M.) \& Trotman (D.) - Scribbling continua in $\mathbb{R}^{n}$ and constructing singularities with prescribed Nash fibre and tangent cone, Topology and Appl., t. 64 (1995), pp. 177-189.

[15] Orro (P.) \& Trotman (D.) - Sur les fibres de Nash de surfaces à singularités isolées, C. R. Acad. Sci. Paris, t. 299 (1984), pp. 397-399.

[16] - On the regular stratifications and conormal structure of subanalytic sets, Bull. London Math. Soc., t. 18 (1986), pp. 185-191.

[17] Cône normal à une stratification régulière, Seminari Geometria 1998-99, Università degli Studi Bologna, t. 12 (2000), pp. 169-175.

[18] TEISSIER (B.) - Variétés polaires II : Multiplicités polaires, sections planes et conditions de Whitney, in Algebraic Geometry, Proc., La Rabida 1981, Lecture Notes in Math., vol. 961, Springer-Verlag, New York, 1982, pp. 314-491.

[19] Trotman (D.) - Counterexamples in stratification theory : two discordant horns, in Real and Complex Singularities, Proceedings 9th Nordic Summer School, Oslo 1976 (Holm (P.), éd.), Sijthoff and Noordhoff, Alphen aan den Rijn, 1977, pp. 679-686.

[20] , Transverse transversals and homeomorphic transversals, Topology, t. 24 (1985), pp. 25-39.

[21] VERDIER (J.-L.) - Stratifications de Whitney et théorème de Bertini-Sard, Invent. Math., t. 36 (1976), pp. 295-312. 